\title{
LII. On striations in the electric discharge
}

\section{Sir J.J. Thomson M.A. F.R.S.}

To cite this article: Sir J.J. Thomson M.A. F.R.S. (1909) LII. On striations in the electric discharge, Philosophical Magazine Series 6, 18:106, 441-451, DOI: 10.1080/14786441008636720

To link to this article: http://dx.doi.org/10.1080/14786441008636720

册 Published online: 21 Apr 2009.

Submit your article to this journal $\pi$

Џll Article views: 7

Q View related articles $\asymp$

4 Citing articles: 22 View citing articles $\asymp$ 
THE

LONDON, EDINBURGH, AND DUBLIN

\title{
PIIILOSOPHICAL MAGAZINE
}

\author{
$A N D$ \\ JOURNAL OF SCIENCE. \\ [SIXTH SERIES.] \\ OCTOBER 1909.
}

LII. On Striations in the Electric Disclarge.

$B y$ Sir J. J. Thomson, M.A., F.R.S.*

NE of the most conspicuous features of the electric discharge through gases, when the pressure is within certain limits, is the exceedingly well-marked alternations of light and darkness which occur in the positive column. These alternatinns, which are called striations, are so varied and beautiful that since their discovery by Abria in 1843 they have attracted the attention of many physicists. Grove, Gassiott, Spottiswoode and Moulton, De la Rue and Müller, Crookes, Wood, Skinner, H. A. Wilson, and Willows have published important researches on the conditions under which the striations are produced; on the influence upon them of such things as the nature and pressure of the gas, the size of the tube, the current passing through it; and on the distribution of the electric force in the neighbourhood of a striation. The investigations described in the following paper relate for the most part to the last of these questions, and were made with the object of testing a theory of the striations which I gave in my Treatise on the Conduction of Electricity through Gases. For these experiments I used tubes fitted with Wehnelt cathodes, $i$. $e$. the cathode was a

* Communicated by the Author. A Discourse given at the Royal Institution on Friday evening, April 2, 1909.

Phil. Mag. S. 6. Vol. 18. No. 106. Oct. 1909. $2 \mathrm{H}$ 
strip of platinum-foil heated to redness, and having on it a spot of lime or barium oxide*.

With these cathodes large currents can be sent through the tube, and remarkably bright and steady striations obtained at lower pressures and with smaller potential-differences than with the ordinary type of discharge. The pressure has, however, to be low, considerably less than $1 \mathrm{~mm}$. of mercury, to get the full advantages from these cathodes. The first point to which attention was directed was the distribution of electric force along the line of the discharge. Investigations on this point have already been made by Skinner and H. A. Wilson, but it seemed to me that the steadiness of the striations with the Wehnelt cathode made this method of investigation particularly suited for these investigations. The first method used to measure the variations in the electric force along the discharge was to find the variation in the difference of potential between two platinum wires $1 \mathrm{~mm}$. apart as the wires were moved from the cathode to the anode. Several devices were used for this purpose: in some the platinum wires (surrounded up to about a millimetre from their tips with glass rods) were carried on a sort of railroad and moved from cathode to anode. The electrodes in the discharge-tube in this case were fixed. The measurements of the potential-differences made by this method at low pressures gave the very remarkable result that just on the cathode side of the bright part of a striation the electric force was negative (i.e. that the force on a positive charge was in the direction from cathode to anode): on crossing over the bright boundary to the anode side the electric force at once became positive, and rose to a high value. It soon, however, began to diminish, and went on diminishing up to the cathode side of the bright part of the next striation on the anode side. The distribution of the electric force in the striation is represented in fig. 1 , and the corresponding distribution of positive and negative electricity in fig. 2, the ordinates representing the distribution of the electrification both as to magnitude and sign. Thus if these measurements of the electric field can be relied on we have intense negative electrification at the bright head of a striation (by head is meant the side next the cathode) and a weak positive electrification through the rest of the field. The

* My assistant, Mr. Everett, has found that these cathodes can be very easily made by letting a drop of sealing-wax fall on the foil, and then burning away the combustible matter by heating the foil to incandescence. Sealing-wax seems to contain large quantities of some salt of barium. 
transition from positive to negative force was very abrupt and well marked, so much so indeed that the position of the

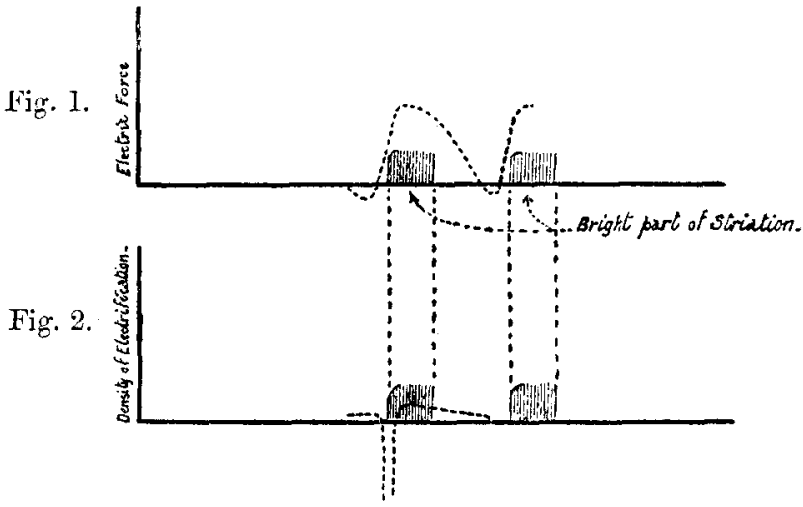

platinum wires in the striation could be ascertained with great accuracy without looking at the discharge, by observing the deflexions of the electrometer by which the potentialdifference between the wires was measured.

Many changes were made in the way in which the wires used as detectors were arranged: thus, to prevent any screening of the one wire by the other, an apparatus was used in which the two platinum wires were brought in from opposite sides of the tube, so that there should be no overlapping; exactly the same results were obtained with this apparatus as with the other. Again, another arrangement, similar to one previously used by Professor H. A. Wilson, was tried, in which the exploring electrodes were kept fixed, and the anode and cathode kept at fixed distance apart were, by means of a float, moved relatively to the exploring electrodes $a, b$, so that these could occupy all positions from the anode to the cathode. The arrangement is represented in fig. 3 (p. 444). The exploring electrodes in some of the experiments protruded about a millimetre beyond the ends of the glass tubes into which. they were sealed; in other experiments very fine hollow glass tubes were used to cover the wires, and the wires instend of protruding beyond the glass stopped short at about a millimetre from the end of the tube; this arrangement was adopted with the idea of protecting the wires against streams of corpuscles coming down the tube: these by giving up their charges to the wire might cause this to acquire potentials different from those of the gas at the tips of the wire. The results obtained with all these modifications were exactly the same as those obtained with the first type of apparatus, $i . e$. there was always when the pressure of the gas was low a $2 \mathrm{H} 2$ 
negative electric force just in front, $i . e$. on the cathode side of the bright part of a striation; this changed to a large

Fig. 3.

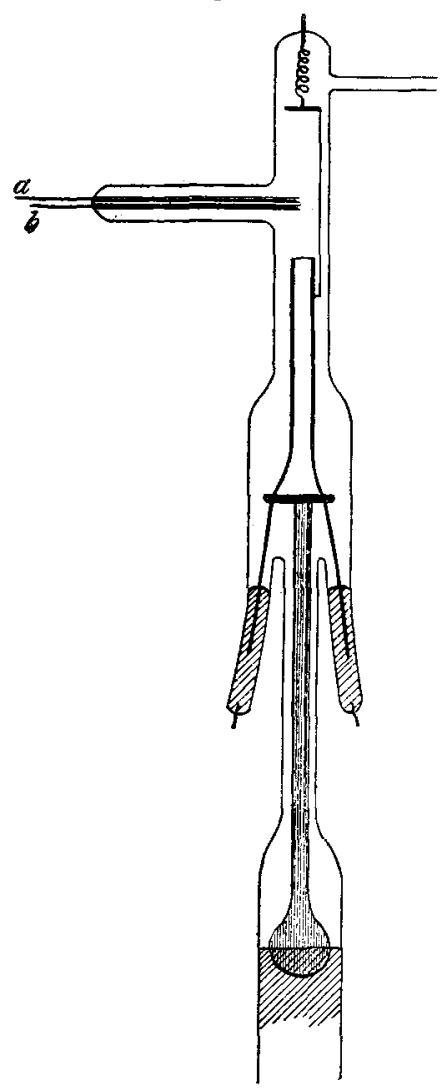

positive force as soon as the bright boundary of the striation was passed; at a short distance from the front of the striation this force began to diminish and went on diminishing until the front of the next striation on the anode side was reached.

Though the indications of all these wire explorers agreed in pointing to the existence of a negative force in front of these striations, yet I felt that the existence of a negative force could never be proved by the use of wire detectors.

For let us consider what the existence of a negative force implies. The electric current is always in the same direction throughout the tube, and therefore the average 
movement of the ions is in the same direction at all parts of the tube; thus, whenever the electric force is negative, there must be ions moving against the electric force instead of with it. Now the validity of the method of the wire electrodes depends upon the assumption that the ions in the neighbourhood of the tip of these electrodes follow the lines of force, that if, for example, the tip were at a higher potential than the gas so that the force on a positive ion were away from the tip, negative ions would follow the direction of the force acting upon them, and run into the tip and lower its potential until it became the same as that of the gas in its neighbourhood. But if the ions do not follow the electric force, and the existence of a negative force implies that some of them at any rate do not, we have no right to assume that the potential of the wire is the same as that of the gas. In some simple cases it is evident that it would not be so. Thus suppose the wire were exposed to a stream of cathode rays, and that there were no positive ions in its neighbourhood, then it is evident that the wire would acquire the potential of the cathode from which the rays started and not that of the gas around the wire.

For these reasons I felt that the existence of a negative force could not be established by means of wire electrodes, and I adopted an entirely different method of measuring the electric force along the discharge-tube. The principle of this method is as follows: imagine a very fine pencil of cathode rays, travelling at right angles to the line joining the cathode and anode, to pass through the discharge-tube. As it crosses the discharge at any place it will be acted upon by the electric force at the point of the discharge, and will be deflected by an amount proportional to the electric force. The deflexion will be from the cathode of the discharge-tube if the force is positive, towards it if the force is negative. If very small pencils of cathode rays are used the disturbance of the electric field in the discharge-tube due to the negative charge on the rays is quite insignificant, and there is none of that distortion of the striations which, to a greater or less extent, always occurs when exploring metallic electrodes are used.

The arrangement by which this principle is carried out in practice is shown in fig. 2. The cathode and anode are fastened together by a piece of glass-rod and fastened to a float, floating on the top of a mercury column. By raising or lowering the column the anode and cathode can be moved up and down the discharge-tube. This arrangement is the same as that used with the wire detectors and shown in fig. 3. 
The wires $a, b$ (fig. 3 ) were replaced by cathode rays generated in the side tube $S$ (fig. 4) by a small induction-coil:

Fig. 4.

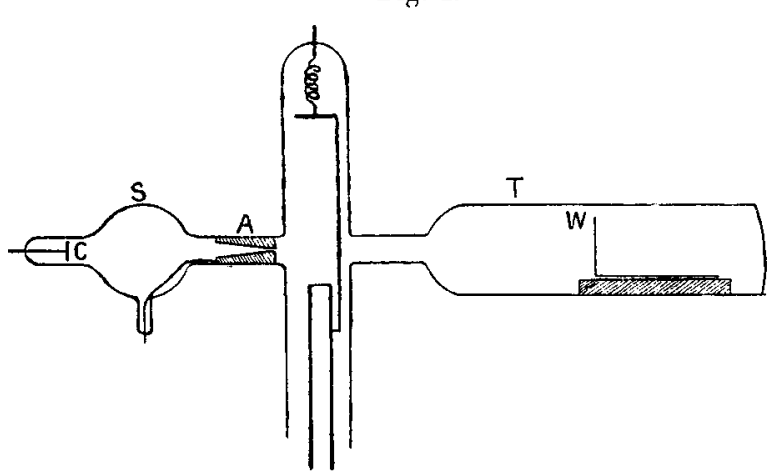

the cathode $\mathrm{C}$ is at the end of the tube, the anode is the metal plug A connected with the earth; a very fine hole was bored in this plug and through it a pencil of rays passed across the discharge and then entered the side tube T. In this tube there was a screen $W$, covered by a phosphorescent substance, in some cases willemite; in others the screen was a zinc sulphide one procured from Mr. Glew. The place of impact of the cathode rays against the screen is marked by a luminous spot, and by measuring with a cathetometer the deflexion of this spot the magnitude and direction of the electric force acting on the cathode rays as they pass across the discharge-tube can be determined. Tinfoil was wrapped round the outside of the discharge-tube to neutralize the effect of electric charges on the glass walls of the tube. The use of cathode rays not only avoids the disturbance due to the presence of the wires, but inasmuch as the cathode rays are negatively electrified particles it enables us to measure the effect of the field on such particles, and as it is the corpuscles which carry practically all the current in the discharge, the method enables us to observe in a very direct way the most important factor in the discharge.

The method is, however, limited to the case where the pressure in the discharge-tube is low, as it is only at low pressures that the cathode rays produce a well-defined spot on the screen.

Observations made with this method showed unmistakably the existence of negative forces in certain parts of the discharge, and in fact the distribution of electric force along 
the tube as determined by this method agreed remarkably well with that determined by the method of the exploring wire. When the discharge was striated it was generally found that where the cathode rays passed underneath a striation, $i . e$. on the catbode side of the bright part of the striation, there was a small deflexion of the cathode rays towards the cathode of the discharge-tube, showing that in this part of the discharge the electric force is negative, while when the path of the cathode rays passed through the bright part of a striation there was a large deflexion of the cathode rays from the cathode of the discharge-tube, showing that in this part of the discharge the electric force was strongly positive. The change from the small negative deflexion to the strong positive one was exceedingly abrupt, so much so that when the anode and cathode were moving downwards, owing to the sinking of the float supporting them, and one striation after another was thus being brought across the path of the cathode rays, the phosphorescent spot moved as abruptly as if it lad been struck by a blow when the bright head of a striation crossed its path. At the low pressures at which these observations are made the potential-difference between the electrodes when the current is large encugh to produce striations is exceedingly small, often not exceeding 60 or 70 volts. Under these circumstances the negative forces although unmistakable are small : when, however, the current through the tube is reduced until the discharge is no longer striated, the potential-difference between the electrodes is very much increased, and now large negative forces can be observed in the neighbourhood of the anode. Sometimes the region in which the force is negative extends a considerable distance from the anode: in one case I observed a negative force for two-thirds of the distance from the anode to the cathode.

As the corpuscles in the cathode rays have an exceedingly small mass they are able to follow very rapid variations in the electric field; by means of them we can observe the gradual establishment of the steady state of the discharge and the change in the direction of the electric force at certain places from positive to negative. Thus suppose the steady current through the tube is small and the potential-difference is considerable, and that the pencil of cathode rays is passing through the discharge near the anode, then if we watch the behaviour of the phosphorescent spot in the interval immediately following the application of the potentialdifference to the tube, we shall find that when the current first starts through the tube the spot is repelled from the cathode, 
showing that at this stage the electric force is positive throughout the tube. This repulsion of the cathode rays is however only momentary : the spot jumps back, and after a very short interval the spot is attracted towards the cathode, showing that the force in this region is now negative. Thus during this interval the ions in the gas and those clinging to the walls of the tube have rearranged themselves in such a way as to reverse the force in the field. This momentary deflexion is much more perceptible near the anode than some distance away from it; the rearrangement seems to spread from the cathode, and to be established so rapidly close to that electrode that there is no time to observe it, while as we travel away from the cathode the steady state is reached after longer and longer intervals, and there is time to observe the initial distribution of the electric field.

We see that the result of the experiments with the cathode rays is to confirm the indications of the wire detector, even when the main current is travelling against the electric force. That the wires in this case should indicate the potential is very remarkable, and must be due I think to the presence in the discharge of slowly moving ions in addition to the swiftly moving ones which carry the main portion of the current, having acquired in other parts of the field sufficient impetus to carry them for some distance against an opposing electric force. The slowly moving ions would be produced by the collisions of the quick ones, and those produced near the tips of the wire electrodes would follow the lines of electric force near the wire and equalize the potentials between the wire and the gas.

The great change in the electric force which occurs at the bright fronts of the striations shows that in these regions we have a great accumulation of negative electricity, while the distribution of the electric force in other parts of the striations and in the dark parts between two striations shows that in those regions there is a slight excess of positive electricity. The magnitude of the charges in the electric force is shown by the following numbers which indicate the electric force in volts per centimetre at different parts of the striation. $x$ in the following table is the distance in millimetres from the bright head of the striation: $x$ is taken positive when measured towards the anode, negative towards the cathode, thus $x=-1$ denotes a place 1 millimetre from the bright head of the striation on the cathode side. $\mathrm{X}$ is the electric force in volts per centimetre at $x$. The gas was hydrogen at a low pressure. 


$\begin{array}{cc}x . & \text { X. } \\ -.5 & -9 \\ +.5 & +67 \\ +1 \cdot 5 & -+33 \\ +3.5 & +30 \\ +7 & +10 \\ +5 & -10\end{array}$

The last reading was at a point just in front of a second striation.

The distance between the bright heads of successivestriations was $9 \mathrm{~mm}$. and the thickness of the dark space $2 \mathrm{~mm}$.

From the preceding table we see that in the space of $1 \mathrm{~mm}$. at the head of a striation we have a change in the electric force of 76 volts per $\mathrm{cm}$. By means of the equation

$$
\frac{d \mathrm{X}}{d x}=4 \pi \rho
$$

we see that the density of the negative electricity at the head of the striation is about $\frac{1}{5}$ of an electrostatic unit per c.c. The density of the positive electricity in the other portions is very much less than this. With Wehnelt electrodes there is frequently only a small potential-difference between corresponding points in adjacent striations: in some cases this difference was only $2 \cdot 7$ volts.

The changes in the electric force are much more abrupt at low pressures than at high ones; though there is always a large increase in the force at the bright head of the striation. I have not observed the existence of the negative forces when the pressure was more than a fraction of a millimetre of mercury.

I have found other cases in which the negative forces are even more pronounced than those I have already considered; perhaps the most striking of these is one where the anode and cathode are connected together and with earth by stout metallic connexions, so that the two are at the same potential, and therefore the average negative force between them is as great as the average positive force. The anodo is perforated by a very fine hole, and through this hole a stream of Canal rays, $i$. e. positively electrified particles, passes into the tube : this produces when the pressure is suitable a fully developed discharge, with striations, Faraday dark space, a well-developed negative glow and dark space; and in spite of the anode and cathode being at the same potential there is in this case the normal cathode fall of about 300 volts at the cathode: the negative forces in a tube of this kind must be very considerable, as they have to balance the cathode fall. 
The heaping up of the negative electricity at the head of the striations seems to me to be the most important factor in the production of striations.

This concentration of the negative electricity at regular intervals along the discharge may be explained as follows. Consider a stream of negative corpuscles projected from the neighbourhood of the cathode with considerable velocity: they will collide against the molecules of the gas, and thereby lose velocity: if the electric field acting on them is not sufficiently intense to restore the velocity lost by the collisions, the corpnscles will lose velocity as they travel through the gas, thus the corpuscles in the rear will gain on those in front, and therefore the density of the corpuscles and therefore of the negative electricity will be greater in the front, and by the equation $\frac{d \mathrm{X}}{d x}=4 \pi \rho$, when $\mathrm{X}$ is the electric force. $x$ the distance from the cathode, and $\rho$ the density of the electricity, the electric force will increase rapidly in consequence of this concentration. This increase in the force will increase the velocity of the particles in front. If the increase in velocity is not sufficient to make the corpuscles ionize the gas by collision, the congestion will be relieved by the gradually increasing velocity of the corpuscles in front, and there will be no periodicity either in the density of the electricity or the electric force. If, however, the force increases so that the corpuscles produce ions by collision quite a different state of affairs will occur; suppose that when the corpuscles get to a place $P$, their velocity is sufficient to produce ionization. On the anode side of $\mathrm{P}$ positive and negative ions will be produced, the positive ones will crowd towards $\mathrm{P}$, the negative ones will move away from it; the consequence will be that there will be an excess of positive electrification on the anode side of $P$ : now positive electrification implies a diminution in the electric force as we move towards the anode, thus the electric force will fall. When it has fallen below the value required for ionization the negative electricity will as before begin to accumulate in the front of the stream, and the electric force will again increase to the ralue required for ionization when the process will be repented. We shall in this way get a periodicity in the electric force such as is observed in the striated discharge. Thus on this view the concare side of the bright head of the striation acts as a cathode, the corresponding anode being the convex side of the bright head of the adjacent striation on the anode side. Between these two places we have a complete discharge, forming a unit by the combination of which the whole discharge is built up. The ions which carry the current through 
any unit are for the most part manufactured in the units themselves, so that these units will behave, as Goldstein and Spottiswoode and Moulton have observed the striations to behave, as if they were to a considerable extent independent of each other. The effect of pressure on the distance between the striations can easily be understood from this point of view, for the lower the pressure the greater will be the distance which particles projected with high velocity will travel before their velocity is destroyed. Again, the variations in the electric field are due to the accumulation of electrical charges in the tube. These accumulations may be regarded as electrified disks whose cross-section is that of the tube; the distance from the disk at which these forces fall to a certain fraction of their maximum value will depend upon the diameter of the disk ; the larger the diameter the greater this distance, so that when the diameter of the tube is small the fluctuations in the intensity of the electric force will be much more rapid than when it is large, and thus we should expect the striations to be much nearer together in a narrow tube than in a wide one.

To explain the variations in the luminosity which accompany these fluctuations in the electric field we must consider the variation in the kinetic energy possessed by the positive ions when they recombine. The recombination of ions does not in general seem to be accompanied by luminosity, unless the ions possess a definite amount of kinetic energy. We certainly can have a gas with great electrical conductivity, and in which a large number of ions are recombining without any visible luminosity; it seems as if the ions must have a definite amount of linetic energy for visible light to be developed on their recombination. Now in the space between two striations the electric field in the part near the cathode side of the bright head of a striation-the dark part-is weak; here the ions have not got the minimum amount of energy requisite for them to be luminous when they recombine; in the bright part of the striation the electric field is strong, and here the ions get sufficient kinetic energy to enable them to give out light when they combine. If the energy required for an ion to give out visible light is greater for light at the blue end of the spectrum than at the red, we might get blue light at one part of the striation, red at another, an effect often observed when we have a mixture of mercury vapour and hydrogen in the tube; a similar separation of the spectra of the two gases in a striation might also be produced if one of the gases were more easily ionized than the other.

I wish to thank my assistant, Mr. Everett, for the assistance he has given me in these investigations. 